www.nature.com/ejhg

\title{
Maternally inherited deafness associated with a T1095C mutation in the mDNA
}

\author{
Alessandro Tessa ${ }^{1,5}$, Aldo Giannotti ${ }^{2}$, Luigi Tieri ${ }^{3}$, Laura Vilarinho ${ }^{5}$, Giacomo Marotta ${ }^{4}$ and \\ Filippo M Santorelli*,1
}

${ }^{1}$ Department of Molecular Medicine, IRCCS-Bambino Gesù Hospital, Rome, Italy; ${ }^{2}$ Department of Genetics, IRCCS-Bambino Gesù Hospital, Rome, Italy; ${ }^{3}$ Department of Audiology, IRCCS-Bambino Gesù Hospital, Rome, Italy; ${ }^{4}$ Az. Osp. Pellegrini, Napoli, Italy; ${ }^{5}$ Department of Clinical Biology, Instituto de Genética Médica, Porto, Portugal

Hearing loss is a relatively frequent defect in children with a genetic or predisposition basis in about $50 \%$ of cases. Mitochondrial DNA (mtDNA)-associated disorder often present with sensorineural hearing loss (SNHL) either in isolation or as a part of a multisystem disorder in adults but the frequency in pediatric cases is unknown. We analysed deafness-related mtDNA mutations in $\mathbf{8 0}$ deaf children to assess the relative frequency of alterations in childhood-onset SNHL. In 16 patients in whom maternal inheritance was possible, we screened for new mutations likely to affect mitochondrial protein synthesis. In one child we detected a novel mutation (T1095C) in the 12S rRNA gene. This mutation fulfils the suggested criteria for definition of a disease-related nucleotide variant. No mutations were found in other patients. Although we cannot exclude the presence of still undefined new mtDNA mutations, our data suggest that mtDNA defect are not common in childhood-onset SNHL. European Journal of Human Genetics (2001) 9, 147-149.

Keywords: mtDNA; hearing loss; mutation

\section{Introduction}

Hearing loss is a relatively frequent sensory defect with approximately one child in 1000 affected and a genetic or predisposition basis in about $50 \%$ of cases. When geneticallydetermined, hearing loss is heterogeneous with an autosomal dominant, recessive, X-linked, or mitochondrial mode of transmission. The exact number of genes involved is unknown, but it is close to 100 , of which only a handful have been identified. ${ }^{1}$ Among these, mutations in the recessive gene GJB2 appear particularly frequent in South European patients. ${ }^{2}$ The final phenotype is, however, the result of an interaction between genetic and environmental factors.

Mitochondrial DNA (mtDNA)-associated disorder often present with sensorineural hearing loss (SNHL) either in isolation or as a part of a multisystem disorder. ${ }^{3}$ About $60 \%$ of

${ }^{*}$ Correspondence: Dr Filippo M Santorelli, Molecular Medicine, IRCCSBambino Gesù Hospital, Piazza S. Onofrio, 4-00165 Rome, Italy. Tel: +39 0668592105; Fax: +39 0668592024; E-mail: fms3@na.flashnet.it. Received 29 June 2000; revised 9 October 2000; accepted 11 October 2000 patients reported with $\mathrm{mtDNA}$ defects have hearing loss and a mutation (A1555G) in the small ribosomal RNA (12S rRNA) is a common cause of maternally inherited SNHL in adults. The mtDNA A3243G mutation is believed associated with as many as $7.4 \%$ of unselected patients with SNHL. ${ }^{4}$ Moreover, the total frequency of mtDNA mutations known to be associated with hearing impairment was shown as $6.9 \%$ in one study. These numbers could be biased by the fact that only frequently reported mtDNA mutations were investigated.

We analysed mtDNA sequences in 80 deaf children to assess the relative frequency of alterations in childhoodonset SNHL. In one child, we detected a novel mutation (T1095C) in the 12S rRNA gene.

\section{Material and methods}

As part of our studies on the genetic factors involved in hearing loss, we analysed 80 children who showed clinical and audiological evidence of non-syndromic hearing loss and no mutations in the whole GJB2 gene. Patients (mean age 7.2 years, range $1-10$ ) were either sporadic or familial cases, the latter with at least two members affected. Mode of 
inheritance was compatible with maternal transmission in 16 cases because of the presence of affected maternal relatives and lack of father-to-son transmission. Patients included individuals with either severe and moderate hearing loss. Clinical characterisation in each patient also used recording of the age at onset of deafness, otoscopic examination, pure tone audiometry, impedance audiometry, and brain-stem auditory evoked responses. The degree of hearing loss was defined according to the mean hearing loss as follows: normal $=<20 \mathrm{~dB}$ hearing loss; mild $=20-40 \mathrm{~dB}$; moderate $=41-70 \mathrm{~dB}$; severe $=71-95 \mathrm{~dB}$; and profound $=>95 \mathrm{~dB}$. Cochlear function was assessed by the presence or absence of transient evoked otoacoustic emissions depending on discernible waves to click stimulation. ${ }^{6}$

Total DNA was extracted from peripheral blood in patients and relatives who agreed to participate in our study and described PCR-mediated site-directed mutagenesis (PSDM) strategies were employed to test for the mtDNA alterations reported in association with syndromic and non-syndromic hearing loss. ${ }^{3,7}$ In particular, we investigated the following mutations: A3243G (in the tRNA ${ }^{\text {Leu(UUR) }}$ gene), A8344G (in the tRNA ${ }^{\text {Lys }}$ gene), A1555G (in the 12S rRNA gene), T8993G (in the ATPase 6 gene), A7445G, 7472insC, and T7511C (in the tRNA $^{\text {Ser(CUN) }}$ gene). In the 16 probands with a possible maternal transmission of the hearing defect and lack of commonly encountered SNHL-associated mtDNA mutations, we also screened for alterations in the 22 tRNAs and the two rRNA genes using single-strand conformation polymorphism (SSCP) and direct sequencing of the abnormally migrating bands.

To screen for the novel T1095C mutation we adopted a PCR-RFLP strategy which employed the endonuclease $H p h \mathrm{I}$ (see legends to figures for details).

\section{Results and Discussion}

None of our patients harboured the above-mentioned mtDNA changes associated with SNHL. One patient had a homoplasmic change $(\mathrm{G}>\mathrm{A})$ at nucleotide position 7444 which is considered a 'secondary' LHON (Leber hereditary optic neuropathy) mutation. However, neither the patient nor his mother and maternal grandmother, who had similar levels of mutated mtDNAs but were not deaf, showed ophthalmologic findings of LHON. SSCP and direct sequencing identified a novel mtDNA mutation (T1095C) in a child who had a strong evidence for a mtDNA defect based on family history (male and female were equally affected in his family, the trait was always transmitted by females, the affected male did not transmit the disorder) and a past medical history of aminoglycoside-induced deafness (AID) in two maternal relatives (I-1 and II-1). Molecular analyses also identified the $\mathrm{T} 1095 \mathrm{C}$ mutation in the three maternal relatives who agreed to undergo genetic testing (Figure 1). This mutation fulfils the suggested criteria for definition of a disease-related nucleotide variant: (1) the mutation, located in the mtDNA $12 \mathrm{~S}$ rRNA gene, occurs at a position that has
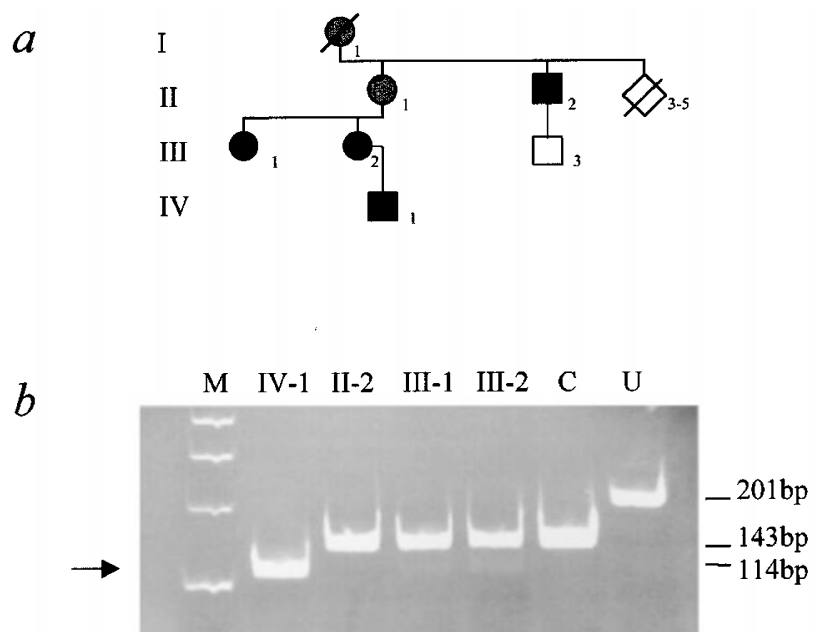

Figure 1 (a) Family pedigree of the family with maternally inherited hearing loss. The black symbols show affected family members and the gray symbols represent individuals with AID, who are reportedly affected but were not directly examined. The white symbols indicate unaffected members. (b) Ethidium bromide-stained gel showing the diagnostic PCR-RFLP analysis used for rapid detection of the novel T1095C mutation (arrow). Patients are as in the family pedigree. C, normal control. $\mathrm{M}$, 100 bp DNA molecular marker. Using oligonucleotide forward primer MT915F 5'-CTCAAGTCAATAGAAGCCGG-3' and reverse primer MT1115R 5'-GGCTGTTGAGGTTTAGGGGT-3' (mismatched nucleotide is underlined), we PCR-amplified a 201 base pairs (bp) fragment. One tenth of the amplicon was cleaved using the endonuclease $\mathrm{Hphl}$ and electrophoresised on a $12 \%$ non-denaturing polyacrylamide gel. The endonuclease $\mathrm{Hphl}$ normally cleaves the 201-bp amplicon into three fragments, sized 143, 31, and $27 \mathrm{bp}$, respectively. The presence of the T1095C mutation introduces an additional site of cleavage, producing fragments sized 114, 31, 29, and 27 bp (not shown). Quantitation of mutated mtDNA used $\left[\alpha_{-}{ }^{32} P\right] d A T P$ in the last PCR cycle. ${ }^{9}$

been highly conserved during evolution (Figure 2); (2) it segregated with the disease in maternal relatives; (3) it was not found in 100 haplotype-matched controls; (4) the mutation was homoplasmic in the proband (>95\%) and less abundant (range $2-45 \%$ ) in maternal relatives.

The remaining 15 patients with a possible maternallyinherited SNHL did not harbour pathogenic mtDNA changes. They had onset of SNHL during early childhood, with a slightly progressive course in nine patients and a sudden onset in six. Impairment of cochlear function was diagnosed only on clinial and audiometric grounds in five patients, and was confirmed by impedance audiometry and brain-stem auditory evoked responses in ten. None of their maternal relatives had other clinical features suggestive of a mtDNA disorder or AID. Although we cannot exclude that these patients (as well as the sporadic cases) still harbour undefined mtDNA mutations, our data suggest that mtDNA defects are not common in childhood-onset SNHL. 

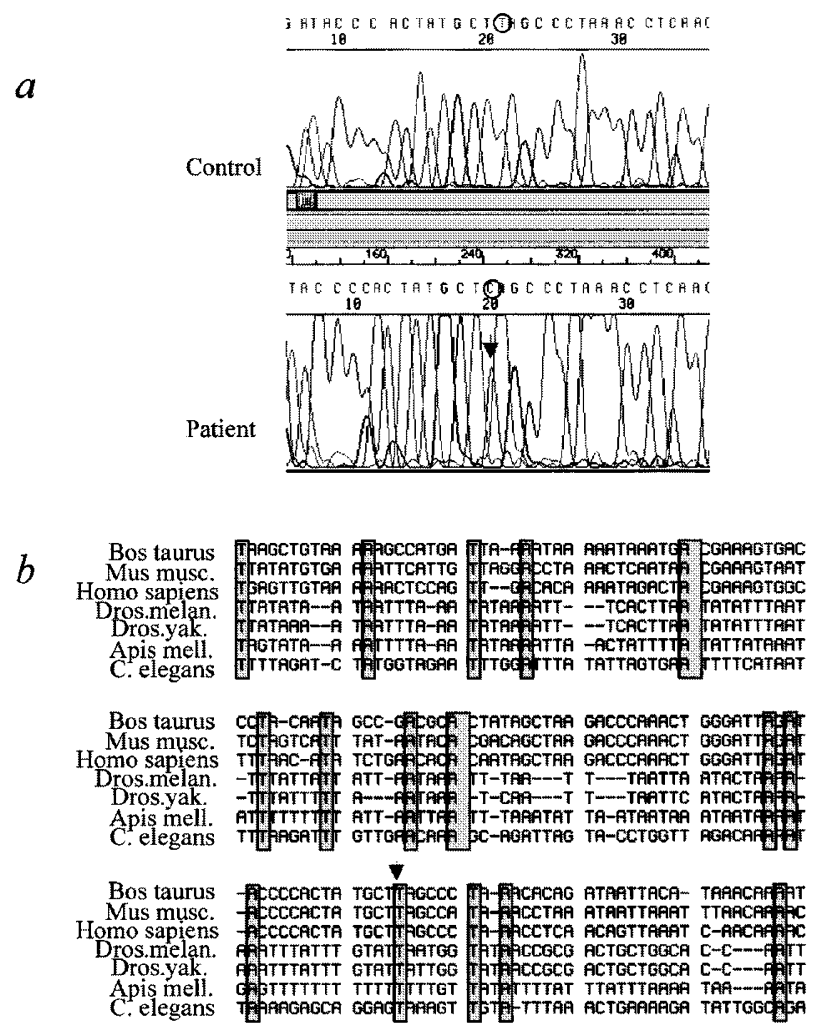

Figure 2 (a) Electropherogram of the 12S rRNA gene flanking the novel missense mutation. The sequence of DNA from a normal control and Patient IV-01 (proband) are presented. Sequencing was performed by means of a sense primer. The novel mutation is indicated (arrow-head). Circles indicate the replaced nucleotide. (b) Sequence alignment of the 12S rRNA gene shows that the T at np1095 (arrow) is highly conserved during evolution.

MtDNA mutations usually affect tissues with high energy requirements, such as muscle and brain. It is reasonable to think that deaf patients harbouring a pathogenic mtDNA mutation might have a central component to their hearing loss. We investigated the characteristics of hearing loss in the family harbouring the T1095C mutation. Patient IV-1 reported a sudden onset of hearing loss. One patient (II-1) had reported a stepwise decline in hearing loss after aminoglycoside treatment while his relatives (II-2, III-1 and III-2) with mild-moderate SNHL noted a slowly progressive loss over many years. Based upon the pure tone audiogram, II-2, III-1 and III-2 had predominantly high frequency hearing loss while IV-1 had a severe/profound hearing loss across all frequencies. Otoacoustic emissions, a sensitive index of cochlear dysfunction, were absent in III-2, with mild hearing loss, and in IV-1 (the proband), with a profound hearing loss. As suggested elsewhere, otoacoustic emissions seem more helpful than evoked response audiometry in establishing whether an individual with moderate or more severe hearing loss and a mtDNA defect has a primarily cochlear defect. ${ }^{8}$ A central contribution to the hearing loss in patients harbouring mtDNA mutations is less likely.

The exact mechanism of cochlear damage in mtDNAassociated disorders is unclear. Normal hearing is dependent upon the hair cells and the stria vascularis, which maintains the ionic gradients necessary for sound signal transduction and the complex interaction between the inner and outer hair cells. Both the stria vascularis and the hair cells are highly metabolically active and would be compromised by a dysfunction of intracellular mitochondrial ATP as a consequence of a mtDNA mutation. ${ }^{3}$ This might ultimately lead to cell death.

We did not observe a straightforward relationship between cochlear dysfunction and mutation load in patients' blood. Although we cannot exclude a higher percentage of mutated mtDNA in the cochlea of patients II-2, III-1, and III-2, it is also possible that environmental factors act synergistically and influence the hearing loss in individuals with a lower mutation load in blood. For instance, this has been hypothesised for the A1555G families in whom aminoglycoside ototoxicity is believed to influence a geneticallydetermined deafness. ${ }^{3}$ This mutation probably makes the human mitochondrial small rRNA structurally more similar to the bacterial rRNA that is the target of aminoglycoside action. Given the history of AID, a similar scenario is also possible for the T1095C family since it occurs in a highly conserved helix close to the $\mathrm{P}$ site of the small mitochondrial ribosomal subunit.

\section{Acknowledgements}

This work was partially supported by the Italian Ministry of Health and a grant from the Italian National Research Council.

\section{References}

1 Willems PJ: Genetic causes of hearing loss. N Engl J Med 2000; 342: $1101-1109$

2 Estivill X, Fortina P, Surrey S et al: Connexin-26 mutations in sporadic and inherited sensorineural deafness. Lancet 1998; 351: 394-398.

3 Fischel-Ghodsian N: Mitochondrial deafness mutations reviewed. Hum Mutat 1999; 13: 261-270.

4 Majamaa K, Moilanen JS, Uimonen S et al: Epidemiology of A3243G, the mutation for mitochondrial encephalomyopathy, lactic acidosis, and strokelike episodes: prevalence of the mutation in an adult population. Am J Hum Genet 1998; 63: $447-454$.

5 Lehtonen MS, Uimonen S, Hassinen IE, Majamaa K: Frequency of mitochondrial DNA point mutations among patients with familial sensorineural hearing impairment. Eur J Hum Genet 2000; 8: 315-318.

6 Martin WH, Schwegler JW, Gleeson AL, Shi YB: New techniques of hearing assessment. Otolaryngol Clin North Am 1994; 27: 487 510.

7 Servidei S: Mitochondrial encephalomyopathies: gene mutation. Neuromusc Disord 1997; 8: XIII-IX.

8 Chinnery P, Elliott C, Green GR et al: The spectrum of hearing loss due to mitochondrial DNA defects. Brain 2000; 123: 82 - 92.

9 Moraes CT, Ricci E, Bonnilla E, DiMauro S, Schon EA: The mitochondrial tRNA ${ }^{\text {Leu(UUR) }}$ mutation in MELAS: genetic, biochemical, and morphological correlations in skeletal muscle. Am J Hum Genet 1992; 50: 934-949. 